$\xi=-1$

\title{
Weibull reliability estimation for $(2+1)$ cascade model
}

\author{
Nada S Karam ${ }^{1 *}$, Ahmed H Khaleel ${ }^{1}$ \\ ${ }^{1}$ College of Education, Department of Mathematics, Almustansiriyah University, Iraq \\ *Corresponding author E-mail: dr_nadaskaram@uomustansiriyah.edu.iq
}

\begin{abstract}
In this paper endeavors to submit reliability $(\mathrm{R})$ of a special $(2+1)$ stress-strength Cascade model for Weibull distribution. Expressions for the model reliability are obtained when the strength and stress are weibull random variables with known shape and unknown scale parameters. Four different methods (ML, Mo, LS and WLS) are used to estimate the reliability and make a comparison between them in simulation study with program made by MATLAB 2016 using criterion MSE, where it found that the best estimator between the four estimators was ML.
\end{abstract}

Keywords: Cascade Model; Reliability; Estimation; Stress-Strength Model; Weibull Distribution.

\section{Introduction}

In a standby system, that the system with standby the redundancy. There are number of the components only one of them works and other remains as standby. The Cascade reliability model is the special type of Strength-Stress model.

Consider a special $(2+1)$ Cascade model with components A, B and $\mathrm{C}$. where the two components $\mathrm{A}$ and $\mathrm{B}$ are activated and the component $\mathrm{C}$ acting as a standby component. Let ' $\mathrm{X}_{1}$ ', ' $\mathrm{X}_{2}$ ' denote the strengths of component (A \& B) respectively and let ' $\mathrm{Y}_{1}$ ', 'Y $\mathrm{Y}_{2}$ ' denote the corresponding stress behaves on them. Here, if failure of any component from the active components A or B the standby component $\mathrm{C}$ is activated. let ' $\mathrm{X}_{3}$ ' be the strength of the component $\mathrm{C}$ and let ' $\mathrm{Y}_{3}$ ' be the stress behaves on it. Here, the component $\mathrm{C}$ will the function with modified the strength $\mathrm{X}_{3}=\mathrm{mX}_{1}\left(\mathrm{mX}_{2}\right)$ and will be influenced by the stress $\mathrm{Y}_{3}=\mathrm{kY}_{1}\left(\mathrm{kY}_{2}\right)$. Here, ' $\mathrm{k}$ ' and ' $\mathrm{m}$ ' denote the stress attenuation factor and strength attenuation factor respectively such that $k>1$ and $0<m<1$.

Gogoi and Borah (2012) [1] deals with the strength vs. stress problem incorporating multi-components system viz. standby redundancy. Sundar (2012) [5] endeavors to present the reliability of an n-cascade system, whose strength and stress distributions are Weibull. Uma Maheswari and Swathi (2013) [6] discussed that reliability of $\mathrm{n}$ - cascade system when stress follows mixed exponential distribution and strength follows exponential distribution. Umamaheswari and Swathi (2013) [7] deals with the generalized exponential distribution with cascade system. Sandhya and Umamaheswari (2013) [3] they work a multi the component standby system of strength-stress model is considered to find reliability, the reliability has been derived when strength-stress follow exponential distribution and the mixture of two exponential distributions. Singh (2013) [4] considered system reliability of ncascade system with strength following exponential distribution and stress following normal distribution. Mutkekar and Munoli (2016) [7] study endeavors to provide the statistical inference for a $(1+1)$ the cascade system for exponential distribution under common effect of strength-stress attenuation factors.

The main aim of this paper is to discuss derivation of mathematical formula of the reliability in special $(2+1)$ Cascade model with strength-stress for weibull distribution by using ML, MO, LS and WLS methods and comparison the results of estimation methods by using the mean square error that will get from the simulation study.

\section{The model reliability}

Let the strength- stress random variables of the three components (two basic and one standby) to be $\mathrm{X}_{\mathrm{i}}$; $\mathrm{i}=1,2,3$ and $\mathrm{Y}_{\mathrm{i}}$; $\mathrm{i}=1,2,3$ respectively are independently and identically distributed Weibull with scale parameter $\beta_{i} ; i=1,2,3$ and scale parameter $\mu_{j} ; j=1,2,3$ and common shape parameter $\alpha$.

The CDF of $\mathrm{W}(\alpha, \beta)$ is:

$F(x)=1-e^{-\beta x^{\alpha}} \quad x>0 ; \alpha, \beta>0$

The PDF of $\mathrm{W}(\alpha, \beta)$ is:

$f(x)=\alpha \beta x^{\alpha-1} e^{-\beta x^{\alpha}} x>0 ; \alpha, \beta>0$

The real reliability function for the $(2+1)$ cascade model is given by:

$$
\begin{aligned}
R= & p\left[X_{1} \geq Y_{1}, X_{2} \geq Y_{2}\right]+p\left[X_{1}<Y_{1}, X_{2} \geq Y_{2}, X_{3} \geq Y_{3}\right] \\
& +\left[X_{1} \geq Y_{1}+X_{2}<Y_{2}, X_{3} \geq Y_{3}\right] \\
R= & R_{1}+R_{2}+R_{3} \\
R_{1}= & p\left[X_{1} \geq Y_{1}, X_{2} \geq Y_{2}\right] \\
= & \int_{0}^{\infty}\left[\bar{F}_{x_{1}}\left(y_{1}\right)\right] g\left(y_{1}\right) d y_{1} \int_{0}^{\infty}\left[\bar{F}_{x_{2}}\left(y_{2}\right)\right] g\left(y_{2}\right) d y_{2}
\end{aligned}
$$

Then will get as

$$
\mathrm{R}_{1}=\left[\frac{\mu_{1}}{\beta_{1}+\mu_{1}}\right]\left[\frac{\mu_{2}}{\beta_{2}+\mu_{2}}\right]
$$

For $\mathrm{R}_{2}$ will begin as: 


$$
\begin{aligned}
\mathrm{R}_{2} & =\mathrm{p}\left[\mathrm{X}_{1}<\mathrm{Y}_{1}, \mathrm{X}_{2} \geq \mathrm{Y}_{2}, \mathrm{X}_{3} \geq \mathrm{Y}_{3}\right] \\
& =\mathrm{p}\left[\mathrm{X}_{1}<\mathrm{Y}_{1}, \mathrm{mX}_{1} \geq \mathrm{kY} \mathrm{Y}_{1}\right] \mathrm{p}\left[\mathrm{X}_{2} \geq \mathrm{Y}_{2}\right]
\end{aligned}
$$

Where

$\mathrm{p}\left[\mathrm{X}_{2} \geq \mathrm{Y}_{2}\right]=\int_{0}^{\infty}\left[\overline{\mathrm{F}}_{\mathrm{x}_{2}}\left(\mathrm{y}_{2}\right)\right] \mathrm{g}\left(\mathrm{y}_{2}\right) \mathrm{dy}_{2}=\left[\frac{\mu_{2}}{\beta_{2}+\mu_{2}}\right]$

And

$$
\begin{aligned}
\mathrm{p}\left[\mathrm{X}_{1}\right. & \left.<\mathrm{Y}_{1}, \mathrm{mX}_{1} \geq \mathrm{k} \mathrm{Y}_{1}\right]=\int_{0}^{\infty}\left[\mathrm{F}_{\mathrm{x}_{1}}\left(\mathrm{y}_{1}\right)\right]\left[\overline{\mathrm{F}}_{\mathrm{x}_{1}}\left(\frac{\mathrm{k}}{\mathrm{m}} \mathrm{y}_{1}\right)\right] \mathrm{g}\left(\mathrm{y}_{1}\right) \mathrm{dy_{1 }} \\
& =\int_{0}^{\infty}\left[1-\mathrm{e}^{-{\beta_{1} \mathrm{y}_{1}{ }^{\alpha_{1}}}}\right]\left[\mathrm{e}^{-\beta_{1}\left(\frac{\mathrm{k}}{\mathrm{m}} \mathrm{y}_{1}\right)^{\alpha_{1}}}\right] \alpha_{1} \mu_{1} \mathrm{y}_{1}{ }^{\alpha_{1}-1} \mathrm{e}^{-\mu_{1} \mathrm{y}_{1}{ }^{\alpha_{1}} \mathrm{dy}} \mathrm{y}_{1} \\
& =\left[\frac{\beta_{1} \mu_{1}}{\left(\left(\frac{\mathrm{k}}{\mathrm{m}}\right)^{\alpha_{1}} \beta_{1}+\mu_{1}\right)\left(\beta_{1}+\left(\frac{\mathrm{k}}{\mathrm{m}}\right)^{\alpha_{1}} \beta_{1}+\mu_{1}\right)}\right]
\end{aligned}
$$

So

$\mathrm{R}_{2}=\left[\frac{\beta_{1} \mu_{1}}{\left(\left(\frac{\mathrm{k}}{\mathrm{m}}\right)^{\alpha_{1}} \beta_{1}+\mu_{1}\right)\left(\beta_{1}+\left(\frac{\mathrm{k}}{\mathrm{m}}\right)^{\alpha_{1}} \beta_{1}+\mu_{1}\right)}\right]\left[\frac{\mu_{2}}{\beta_{2}+\mu_{2}}\right]$

Similarly for $\mathrm{R}_{3}$ :

$$
\begin{aligned}
R_{3} & =\left[X_{1} \geq Y_{1}+X_{2}<Y_{2}, X_{3} \geq Y_{3}\right] \\
& =\left[\frac{\mu_{1}}{\beta_{1}+\mu_{1}}\right]\left[\frac{\beta_{2} \mu_{2}}{\left(\left(\frac{k}{m}\right)^{\alpha_{2}} \beta_{2}+\mu_{2}\right)\left(\beta_{2}+\left(\frac{k}{m}\right)^{\alpha_{2}} \beta_{2}+\mu_{2}\right)}\right]
\end{aligned}
$$

Substitution (4), (5) and (6) in (3) say; R

$$
\begin{aligned}
\mathrm{R}= & {\left[\frac{\mu_{1}}{\beta_{1}+\mu_{1}}\right]\left[\frac{\mu_{2}}{\beta_{2}+\mu_{2}}\right]+\left[\frac{\beta_{1} \mu_{1}}{\left(\left(\frac{\mathrm{k}}{\mathrm{m}}\right)^{\alpha_{1}} \beta_{1}+\mu_{1}\right)\left(\beta_{1}+\left(\frac{\mathrm{k}}{\mathrm{m}}\right)^{\alpha_{1}} \beta_{1}+\mu_{1}\right)}\right]\left[\frac{\mu_{2}}{\beta_{2}+\mu_{2}}\right] } \\
& +\left[\frac{\mu_{1}}{\beta_{1}+\mu_{1}}\right]\left[\frac{\beta_{2} \mu_{2}}{\left(\left(\frac{\mathrm{k}}{\mathrm{m}}\right)^{\alpha_{2}} \beta_{2}+\mu_{2}\right)\left(\beta_{2}+\left(\frac{\mathrm{k}}{\mathrm{m}}\right)^{\alpha_{2}} \beta_{2}+\mu_{2}\right)}\right]
\end{aligned}
$$

\section{The model reliability estimation}

\section{1. Maximum likelihood estimation method (ML)}

Let $X_{i} ; i=1,2,3$ strength random sample have $W(\alpha, \beta)$ distribution with the sample size $n$, where $\alpha$ is known shape parameter and $\beta$ is unknown scale parameter, then the maximum likelihood function $\mathrm{L}$; the probability joint function; with general form will be as:

$L\left(x_{1}, x_{2}, \ldots x_{n}, \alpha, \beta\right)=(\alpha \beta)^{n} \prod_{i=1}^{n} x_{i}^{\alpha-1} e^{-\beta \sum_{i=1}^{n} x_{i}^{\alpha}}$

Taken the natural logarithm for function in the equation (8):

$\operatorname{LnL}=n \operatorname{Ln} \alpha+n \operatorname{Ln} \beta+(\alpha-1) \sum_{\mathrm{i}=1}^{\mathrm{n}} \ln \mathrm{x}_{\mathrm{i}}-\beta \sum_{\mathrm{i}=1}^{\mathrm{n}} \mathrm{x}_{\mathrm{i}}^{\alpha}$

Taken the partial derivative to equation (9) with respect of unknown parameter $\beta$, then we get:

$\frac{\partial \ln L}{\partial \beta}=\frac{n}{\beta}-\sum_{i=1}^{n} X_{i}^{\alpha}$

Equating the equation (10) to zero, then the maximum likelihood estimator for $\beta$ :

$\hat{\beta}_{(\mathrm{ML})}=\frac{\mathrm{n}}{\sum_{\mathrm{i}=1}^{\mathrm{n}} \mathrm{x}_{\mathrm{i}}^{\alpha}}$

And let $\mathrm{X}_{1_{\mathrm{i}_{1}}} ; \mathrm{i}_{1}=1,2, \ldots, \mathrm{n}_{1} \mathrm{X}_{2_{\mathrm{i}_{2}}} ; \mathrm{i}_{2}=1,2, \ldots, \mathrm{n}_{2}$ and $\mathrm{X}_{3_{\mathrm{i}_{3}}} ; \mathrm{i}_{3}=$ $1,2, \ldots, n_{3}$ strength random samples from $W\left(\alpha_{1}, \beta_{1}\right), W\left(\alpha_{2}, \beta_{2}\right)$ and $\mathrm{W}\left(\alpha_{3}, \beta_{3}\right)$, with samples size $\mathrm{n}_{1}, \mathrm{n}_{2}$ and $\mathrm{n}_{3}$ respectively where $\beta_{1}, \beta_{2}$ and $\beta_{3}$ are unknown parameters:

$\widehat{\beta}_{\xi(\mathrm{ML})}=\frac{\mathrm{n}_{\xi}}{\sum_{\mathrm{i}_{\xi}=1}^{\mathrm{n}_{\xi} \mathrm{x}_{\xi} \mathrm{i}_{\xi}}}, \xi=1,2,3$

For the stress random variables the ML estimator for unknown parameters $\mu_{1}, \mu_{2}$ and $\mu_{3}$ will be as:

$\hat{\mu}_{\xi(\mathrm{ML})}=\frac{\mathrm{m}_{\xi}}{\sum_{\mathrm{j}_{\xi}=1}^{\mathrm{m}_{\xi} \mathrm{y}_{\xi} \mathrm{j}_{\xi}}}, \xi=1,2,3$

Substitution (11) and (12) in (7) the ML estimator for reliability say; $\widehat{\mathrm{R}}_{(\mathrm{ML})}$; invariability will be as:

$$
\begin{aligned}
& \widehat{\mathrm{R}}_{(\mathrm{ML})}=\left[\frac{\widehat{\mu}_{1(\mathrm{ML})}}{\widehat{\hat{\beta}}_{1(\mathrm{ML})}+\widehat{\mu}_{1(\mathrm{ML})}}\right]\left[\frac{\widehat{\mu}_{2(\mathrm{ML})}}{\hat{\beta}_{2(\mathrm{ML})}+\widehat{\mu}_{2(\mathrm{ML})}}\right]
\end{aligned}
$$

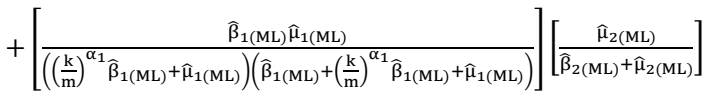

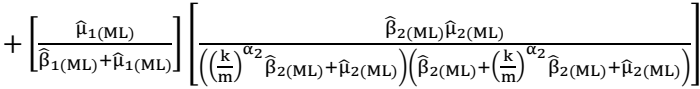

\subsection{Moment estimation method (Mo)}

In the method of moment first step we need the mean population of $\mathrm{W}(\alpha, \beta)$ :

$$
\mathrm{E}(\mathrm{x})=\frac{1}{\beta^{\frac{1}{\alpha}}} \Gamma\left(1+\frac{1}{\alpha}\right)
$$

Second step equating the mean sample with corresponding mean population, then we get the unknown scale parameter $\beta$ moment estimators

$$
\frac{\sum_{\mathrm{i}=1}^{\mathrm{n}} \mathrm{x}_{\mathrm{i}}}{\mathrm{n}}=\frac{1}{\beta^{\frac{1}{\alpha}}} \Gamma\left(1+\frac{1}{\alpha}\right)
$$

Then we get the moment estimator of $\beta$ is:

$\hat{\beta}_{(\mathrm{Mo})}=\left[\frac{\Gamma\left(1+\frac{1}{\alpha}\right)}{\overline{\mathrm{x}}}\right]^{\alpha}$

Will get as

$\widehat{\beta}_{\xi(\mathrm{Mo})}=\left[\frac{\Gamma\left(1+\frac{1}{\alpha_{\xi}}\right)}{\overline{\mathrm{x}}_{\xi}}\right]^{\alpha_{\xi}}, \xi=1,2,3$

And

$\hat{\mu}_{\xi(\mathrm{Mo})}=\left[\frac{\Gamma\left(1+\frac{1}{\alpha_{\xi}}\right)}{\overline{\mathrm{y}} \xi}\right]^{\alpha_{\xi}}, \xi=1,2,3$

Substitution (17) and (18) in (7) estimator for reliability say; $\widehat{\mathrm{R}}_{(\mathrm{Mo})}$; approximately will be as:

$$
\begin{aligned}
& \widehat{\mathrm{R}}_{(\mathrm{Mo})}=\left[\frac{\widehat{\mu}_{1(\mathrm{Mo})}}{\widehat{\hat{\beta}}_{1(\mathrm{Mo})}+\widehat{\mu}_{1(\mathrm{Mo})}}\right]\left[\frac{\widehat{\mu}_{2(\mathrm{Mo})}}{\widehat{\hat{\beta}}_{2(\mathrm{Mo})}+\widehat{\mu}_{2(\mathrm{Mo})}}\right]
\end{aligned}
$$

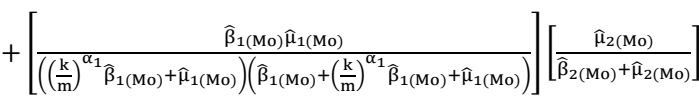

$$
\begin{aligned}
& +\left[\frac{\hat{\mu}_{1(\mathrm{Mo})}}{\widehat{\hat{\beta}}_{1(\mathrm{Mo})}+\widehat{\mu}_{1(\mathrm{Mo})}}\right]\left[\frac{\widehat{\beta}_{2(\mathrm{Mo}) \hat{\mu}_{2(\mathrm{Mo})}}}{\left(\left(\frac{\mathrm{k}}{\mathrm{m}}\right)^{\alpha_{2}} \widehat{\hat{\beta}}_{2(\mathrm{Mo})}+\hat{\mu}_{2(\mathrm{Mo})}\right)\left(\widehat{\hat{\beta}}_{2(\mathrm{Mo})}+\left(\frac{\mathrm{k}}{\mathrm{m}}\right)^{\alpha_{2}} \widehat{\hat{\beta}}_{2(\mathrm{Mo})}+\hat{\mu}_{2(\mathrm{Mo})}\right)}\right]
\end{aligned}
$$




\section{3. Least square estimation method (LS)}

We can use method of least square for estimate the parameters of the Weibull distribution by minimizing equation:

$$
\mathrm{S}=\sum_{\mathrm{i}=1}^{\mathrm{n}}\left[\mathrm{F}\left(\mathrm{X}_{(\mathrm{i})}\right)-\mathrm{E}\left(\mathrm{F}\left(\mathrm{X}_{(\mathrm{i})}\right)\right)\right]^{2}
$$

Where $E\left(F\left(X_{(i)}\right)\right)$ equal to the plotting position $P_{i}$, and $P_{i}=\frac{i}{n+1}$

Taking natural logarithm to the $\left(1-\mathrm{P}_{\mathrm{i}}\right)=\mathrm{e}^{-\beta \mathrm{x}_{(\mathrm{i})}^{\alpha}}$ we get:

$$
\ln \left(1-P_{i}\right)+\beta x_{(i)}^{\alpha}=0
$$

Substitution (22) in (21) we get:

$\mathrm{S}=\sum_{\mathrm{i}=1}^{\mathrm{n}}\left[\ln \left(1-\mathrm{P}_{\mathrm{i}}\right)+\beta \mathrm{x}_{(\mathrm{i})}^{\alpha}\right]^{2}$

Deriving (23) with respect to the unknown scale parameter $\beta$ and equating result to the zero, then we will get:

$$
\begin{aligned}
& \frac{\partial \mathrm{S}}{\partial \beta}=\sum_{\mathrm{i}=1}^{\mathrm{n}} 2\left[\ln \left(1-\mathrm{P}_{\mathrm{i}}\right)+\beta \mathrm{x}_{(\mathrm{i})}^{\alpha}\right] \mathrm{x}_{(\mathrm{i})}^{\alpha} \\
& \sum_{\mathrm{i}=1}^{\mathrm{n}} \mathrm{x}_{(\mathrm{i})}^{\alpha} \ln \left(1-\mathrm{P}_{\mathrm{i}}\right)+\beta \sum_{\mathrm{i}=1}^{\mathrm{n}} \mathrm{x}_{(\mathrm{i})}^{2 \alpha}=0
\end{aligned}
$$

Then we get the estimator of $\beta$ is:

$$
\widehat{\beta}_{(L S)}=\frac{-\sum_{\mathrm{i}=1}^{\mathrm{n}} \mathrm{x}_{(\mathrm{i})}^{\alpha} \ln \left(1-\mathrm{P}_{\mathrm{i}}\right)}{\sum_{\mathrm{i}=1}^{\mathrm{n}} \mathrm{x}_{(\mathrm{i})}^{2 \alpha}}
$$

Will get as

$$
\widehat{\beta}_{\xi(\mathrm{LS})}=\frac{-\sum_{\mathrm{i}_{\xi}=1}^{\mathrm{n}_{\xi} \mathrm{x}_{\xi}}\left(\mathrm{i}_{\xi}\right)}{\ln \left(1-\mathrm{P}_{\mathrm{i}}\right)}, \xi=1,2,3
$$

And

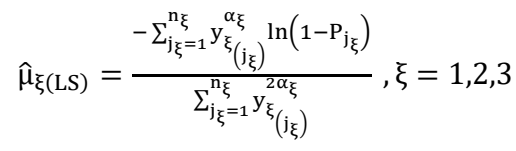

Where $P_{j}=\frac{j}{m+1}, j=1,2, \ldots, m$

Substitution (26) and (27) in (7) estimator for reliability say; $\widehat{\mathrm{R}}_{(\mathrm{LS})}$ ; approximately will be as:

$$
\begin{aligned}
& \widehat{\mathrm{R}}_{(\mathrm{LS})}=\left[\frac{\widehat{\mu}_{1(\mathrm{LS})}}{\widehat{\hat{\beta}}_{1(\mathrm{LS})}+\widehat{\mu}_{1(\mathrm{LS})}}\right]\left[\frac{\widehat{\mu}_{2(\mathrm{LS})}}{\widehat{\hat{\beta}}_{2(\mathrm{LS})}+\hat{\mu}_{2(\mathrm{LS})}}\right] \\
& +\left[\frac{\widehat{\beta}_{1(L S)} \hat{\mu}_{1(L S)}}{\left(\left(\frac{k}{m}\right)^{\alpha_{1}} \widehat{\beta}_{1(L S)}+\widehat{\mu}_{1(L S)}\right)\left(\widehat{\beta}_{1(L S)}+\left(\frac{k}{m}\right)^{\alpha_{1}} \widehat{\beta}_{1(L S)}+\widehat{\mu}_{1(L S)}\right)}\right]\left[\frac{\hat{\mu}_{2(L S)}}{\hat{\beta}_{2(L S)}+\widehat{\mu}_{2(L S)}}\right] \\
& +\left[\frac{\widehat{\mu}_{1(L S)}}{\widehat{\beta}_{1(L S)}+\widehat{\mu}_{1(L S)}}\right]\left[\frac{\widehat{\beta}_{2(L S)} \widehat{\mu}_{2(L S)}}{\left(\left(\frac{k}{m}\right)^{\alpha_{2}} \widehat{\beta}_{2(L S)}+\hat{\mu}_{2(L S)}\right)\left(\widehat{\beta}_{2(L S)}+\left(\frac{k}{m}\right)^{\alpha_{2}} \widehat{\hat{\beta}}_{2(L S)}+\hat{\mu}_{2(L S)}\right)}\right]
\end{aligned}
$$

\section{4 Weighted least square estimation method (WLS)}

We can use method of weighted least squares estimators of the Weibull distribution by minimizing the following equation:

$$
\mathrm{S}=\sum_{\mathrm{i}=1}^{\mathrm{n}} \mathrm{w}_{\mathrm{i}}\left[\mathrm{F}\left(\mathrm{x}_{(\mathrm{i})}\right)-\mathrm{E}\left(\mathrm{F}\left(\mathrm{x}_{(\mathrm{i})}\right)\right)\right]^{2}
$$

Where $\mathrm{w}_{\mathrm{i}}=\frac{1}{\operatorname{Var}\left[\mathrm{F}\left(\mathrm{x}_{\mathrm{i}}\right)\right]}=\frac{(\mathrm{n}+1)^{2}(\mathrm{n}+2)}{\mathrm{i}(\mathrm{n}-\mathrm{i}+1)}, \mathrm{i}=1,2, \ldots, \mathrm{n}$

As in equation (22) we get:

$$
S=\sum_{i=1}^{n} w_{i}\left[\ln \left(1-P_{i}\right)+\beta x_{(i)}^{\alpha}\right]^{2}
$$

Deriving (30) with respect to the scale parameter $\beta$ and equating result to the zero, then we will get:

$\frac{\partial S}{\partial \beta}=\sum_{i=1}^{n} 2 w_{i}\left[\ln \left(1-P_{i}\right)+\beta x_{(i)}^{\alpha}\right] x_{(i)}^{\alpha}$

$\sum_{\mathrm{i}=1}^{\mathrm{n}} \mathrm{w}_{\mathrm{i}} \mathrm{x}_{(\mathrm{i})}^{\alpha}\left(1-\mathrm{P}_{\mathrm{i}}\right)+\beta \sum_{\mathrm{i}=1}^{\mathrm{n}} \mathrm{w}_{\mathrm{i}} \mathrm{x}_{(\mathrm{i})}^{2 \alpha}=0$

Then we get the estimator of $\beta$ is:

$\widehat{\beta}_{(W L S)}=\frac{-\sum_{\mathrm{i}=1}^{\mathrm{n}} \mathrm{w}_{\mathrm{i}} \mathrm{x}_{(\mathrm{i})}^{\alpha} \ln \left(1-\mathrm{P}_{\mathrm{i}}\right)}{\sum_{\mathrm{i}=1}^{\mathrm{n}} \mathrm{w}_{\mathrm{i}} \mathrm{x}_{(\mathrm{i})}^{2 \alpha}}$

Will get as

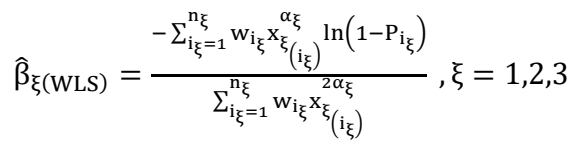

And

$\hat{\mu}_{\xi(\mathrm{WLS})}=\frac{-\sum_{\mathrm{j}_{\xi}=1}^{\left.\mathrm{n}_{\xi} \mathrm{w}_{\mathrm{j} \xi} \mathrm{y}_{\xi} \mathrm{d}_{(\mathrm{j}}\right)} \ln \left(1-\mathrm{P}_{\mathrm{j} \xi}\right)}{\sum_{\mathrm{j}_{\xi}=1}^{\mathrm{n}_{\xi}} \mathrm{w}_{\mathrm{j}_{\xi}} \mathrm{y}_{\xi} \mathrm{y}_{\xi}}, \xi=1,2,3$

Where $w_{j}=\frac{1}{\operatorname{Var}\left[G\left(y_{(j)}\right)\right]}=\frac{(m+1)^{2}(m+2)}{j(m-j+1)}, j=1,2, \ldots, m$

Substitution (33) and (34) in (7) estimator for reliability say; $\widehat{\mathrm{R}}_{(\mathrm{WLS})}$; approximately will be as:

$\widehat{\mathrm{R}}_{(\mathrm{WLS})}=\left[\frac{\widehat{\mu}_{1(\mathrm{WLS})}}{\widehat{\hat{\beta}}_{1(\mathrm{WLS})}+\widehat{\mu}_{1(\mathrm{WLS})}}\right]\left[\frac{\widehat{\mu}_{2(\mathrm{WLS})}}{\widehat{\hat{\beta}}_{2(\mathrm{WLS})}+\widehat{\mu}_{2(\mathrm{WLS})}}\right]$

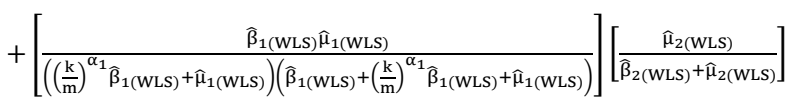

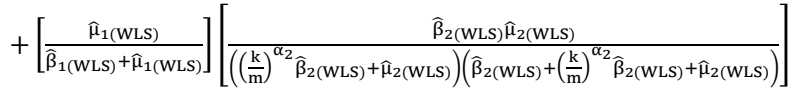

\section{Estimators comparison}

The simulation study will be advanced to show estimators behavior of $\mathrm{R}$ by different four estimation methods then compare results by using the statistical criteria mean square error (MSE). The simulation study is repeated (10000) times to obtain independent the samples of different sizes.

\section{1. Generating random variables}

Let $\mathrm{U}$ be a random variable with the uniform distribution in $(0,1)$ then data for the Weibull distributions can be generated by adoption of the inverse transformation for the CDF where if:

$\mathrm{U}=\mathrm{F}(\mathrm{X}) \rightarrow \mathrm{X}=\mathrm{F}^{-1}(\mathrm{U})$

Then

$X=\left(-\frac{1}{\beta} \ln (1-U)\right)^{\frac{1}{\alpha}}$

$\mathrm{X}_{\mathrm{i}_{\xi}}=\left(-\frac{1}{\beta_{\mathrm{i} \xi}} \ln \left(1-\mathrm{U}_{\mathrm{i}_{\xi}}\right)\right)^{\frac{1}{\alpha_{\mathrm{i} \xi}}} ; \mathrm{i}=1,2, \ldots, \mathrm{n}_{\xi} ; \xi=1,2,3$ 
And

$Y_{j_{\xi}}=\left(-\frac{1}{\mu_{j \xi}} \ln \left(1-U_{j_{\xi}}\right)\right)^{\frac{1}{\alpha_{\mathrm{j} \xi}}} \mathrm{j}=1,2, \ldots, \mathrm{m}_{\xi} ; \xi=1,2,3$

\section{2. Simulation study}

The simulation program is written by using the MATLAB 2016 to make the comparison between the reliability estimators, which can be described through following the steps:

1) A random samples $x_{11}, x_{12}, \ldots, x_{1 n_{1}}, x_{21}, x_{22}, \ldots, x_{2 n_{2}}$, and $\mathrm{y}_{11}, \mathrm{y}_{12}, \ldots, \mathrm{y}_{1 \mathrm{~m}_{1}}, \mathrm{y}_{21}, \mathrm{y}_{22}, \ldots, \mathrm{y}_{2 \mathrm{~m}_{2}}$ of sizes

$\left(\mathrm{n}_{1}, \mathrm{n}_{2}, \mathrm{n}_{1}, \mathrm{n}_{2}\right)=(10,10,10,10),(25,25,25,25),(75,75,75,75)$ and $(10,25,75,75)$ are generated from Weibull distributions.

2) The real parameters values are selected for 6 experiments $\left(\alpha_{1}, \alpha_{2}, \beta_{1}, \beta_{2}, \mu_{1}, \mu_{2}\right)$ in table (1)

Table 1: The Parameters Values

\begin{tabular}{cccccccccc}
\hline Exp. & $\mathrm{k}$ & $\mathrm{m}$ & $\alpha_{1}$ & $\alpha_{2}$ & $\beta_{1}$ & $\beta_{2}$ & $\mu_{1}$ & $\mu_{2}$ & $\mathrm{R}$ \\
\hline 1 & 2 & 0.2 & 2 & 2 & 2 & 2 & 2 & 2 & 0.3648 \\
2 & 2 & 0.2 & 2 & 2 & 3 & 3 & 2 & 2 & 0.1601 \\
3 & 1.6 & 0.4 & 2 & 2 & 3 & 3 & 2 & 2 & 0.1618 \\
4 & 1.6 & 0.4 & 2 & 2 & 2 & 2 & 3 & 3 & 0.3656 \\
5 & 1.1 & 0.9 & 2 & 2 & 2 & 2 & 3 & 3 & 0.5105 \\
6 & 1.1 & 0.9 & 4 & 4 & 3 & 3 & 3 & 3 & 0.3231 \\
\hline
\end{tabular}

3) The parameters $\beta_{1}, \beta_{2}, \mu_{1}, \mu_{2}$ are estimated by (ML, MO LS, and WLS) as in the equations: (12), (13), (18), (19), (26), (27), (33) and (34) respectively.

4) The estimation of $\mathrm{R}$ is estimated as in the equations: (14) (20), (28) and (35).

5) Compute the mean by the equation:

Mean $=\frac{\sum_{\mathrm{i}=1}^{\mathrm{L}} \widehat{\mathrm{R}}_{\mathrm{i}}}{\mathrm{L}}$

6) After finding estimators in previous steps, at this step the comparison between the four estimation methods, is done by using the Mean square error.

The formula is: $\operatorname{MSE}(\widehat{\mathrm{R}})=\frac{1}{\mathrm{~L}} \sum_{\mathrm{i}=1}^{\mathrm{L}}\left(\widehat{\mathrm{R}}_{\mathrm{i}}-\mathrm{R}\right)^{2}$

\section{3. Simulation models}

After applying the previous steps of $\mathrm{R}$ for sample size $\left(\mathrm{n}_{1}, \mathrm{n}_{2}, \mathrm{~m}_{1}, \mathrm{~m}_{2}\right)=\mathrm{a}, \mathrm{b}, \mathrm{c}$ and $\mathrm{d}$ where $\mathrm{a}=(10,10,10,10), \mathrm{b}=$ $(25,25,25,25), c=(75,75,75,75)$ and $d=(10,25,75,75)$ respectively for table values (1):

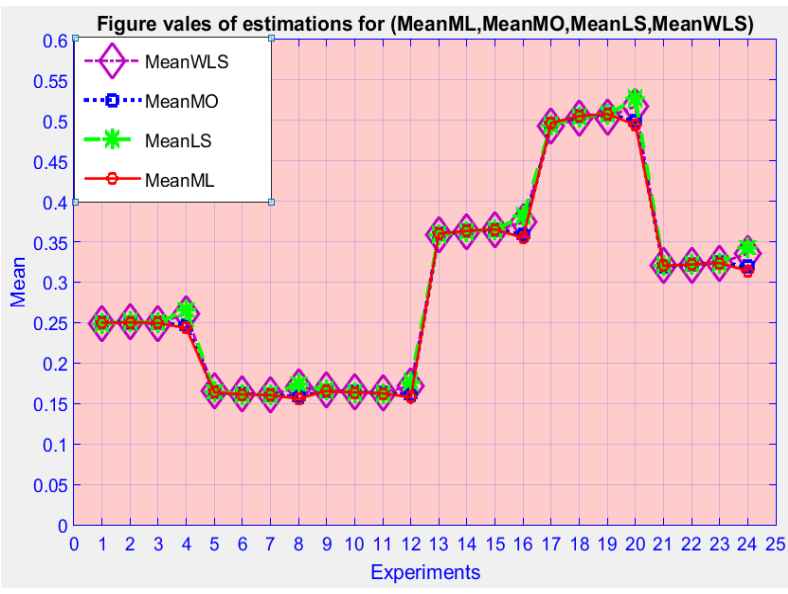

Fig. 1: Represent the Mean of (ML, MO, LS, WLS) Estimators.

Table 2: The MSE Values for Experiment (1)

\begin{tabular}{ccccc}
\multicolumn{5}{c}{ Table 2: The MSE Values for Experiment (1) } \\
\hline Simple size & ML & Mo & LS & WLS \\
\hline a & 0.0060 & 0.0066 & 0.0067 & 0.0074 \\
b & 0.0025 & 0.0027 & 0.0031 & 0.0041 \\
c & 0.0008 & 0.0009 & 0.0010 & 0.0020 \\
d & 0.0026 & 0.0028 & 0.0035 & 0.0043 \\
\hline
\end{tabular}

Table3: The MSE Values for Experiment (2)

\begin{tabular}{|c|c|c|c|c|}
\hline Simple size & $\mathrm{ML}$ & Mo & LS & WLS \\
\hline $\mathrm{a}$ & 0.0039 & 0.0041 & 0.0045 & 0.0050 \\
\hline $\mathrm{b}$ & 0.0015 & 0.0016 & 0.0018 & 0.0024 \\
\hline $\mathrm{c}$ & 0.0004 & 0.0005 & 0.0006 & 0.0012 \\
\hline d & 0.0015 & 0.0017 & 0.0022 & 0.0027 \\
\hline
\end{tabular}

Table 4: The MSE Values for Experiment (3)

\begin{tabular}{ccccc}
\multicolumn{5}{c}{ Table 4: The MSE Values for Experiment (3) } \\
\hline Simple size & ML & Mo & LS & WLS \\
\hline a & 0.0039 & 0.0042 & 0.0045 & 0.0051 \\
b & 0.0015 & 0.0017 & 0.0019 & 0.0026 \\
c & 0.0005 & 0.0007 & 0.0006 & 0.0013 \\
d & 0.0016 & 0.0017 & 0.0023 & 0.0028 \\
\hline
\end{tabular}

\begin{tabular}{ccccc}
\multicolumn{5}{c}{ Table 5: The MSE Values for Experiment (4) } \\
\hline Simple size & ML & Mo & LS & WLS \\
\hline a & 0.0083 & 0.0089 & 0.0095 & 0.0106 \\
b & 0.0035 & 0.0038 & 0.0042 & 0.0057 \\
c & 0.0011 & 0.0012 & 0.0014 & 0.0029 \\
d & 0.0037 & 0.0040 & 0.0046 & 0.0057 \\
\hline
\end{tabular}

Table 6: The MSE Values for Experiment (5)

\begin{tabular}{ccccc}
\hline Simple size & ML & Mo & LS & WLS \\
\hline $\mathrm{a}$ & 0.0128 & 0.0139 & 0.0144 & 0.0160 \\
$\mathrm{~b}$ & 0.0053 & 0.0057 & 0.0065 & 0.0087 \\
$\mathrm{c}$ & 0.0017 & 0.0019 & 0.0022 & 0.0044 \\
$\mathrm{~d}$ & 0.0060 & 0.0063 & 0.0069 & 0.0086 \\
\hline
\end{tabular}

Table 7: The Msevalues for Experiment (6)

\begin{tabular}{ccccc}
\multicolumn{5}{c}{ Table 7: The Msevalues for Experiment (6) } \\
\hline Simple size & ML & Mo & LS & WLS \\
\hline a & 0.0097 & 0.0116 & 0.0110 & 0.0122 \\
b & 0.0041 & 0.0050 & 0.0049 & 0.0066 \\
c & 0.0014 & 0.0017 & 0.0017 & 0.0034 \\
d & 0.0043 & 0.0052 & 0.0056 & 0.0068 \\
\hline
\end{tabular}

\section{Conclusions}

These conclusions are made according to simulation results that have been compared in the previous steps:

A. From table (1) we concluded the following:

1) When the value of the common shape parameter $\alpha$ is increases, the reliability is decreases.

2) When the value of the scale parameter $\beta$ is increases, the Reliability is decreases.

3) When the value of scale parameter $\mu$ is increases, the Reliability is increases.

4) When the value of the $\frac{\mathrm{k}}{\mathrm{m}}$ decreases (where ' $m$ ' and ' $k$ ' denote the strength attenuation factor and the stress attenuation factor) the Reliability is increases.

B. The best estimation method of MSE for R:

\begin{tabular}{lr}
\hline \multicolumn{1}{c}{ Sample size and parameters value } & Best method \\
\hline For $\left(\mathrm{n}_{1}, \mathrm{n}_{2}, \mathrm{~m}_{1}, \mathrm{~m}_{2}\right)=(10,10,10,10),(25,25,25,25)$, & \\
$(75,75,75,75)$, and $(10,25,75,75)$ when & \\
$\left(\alpha_{1}, \alpha_{2}, \beta_{1}, \beta_{2}, \mu_{1}, \mu_{2}\right)=(2,2,2,2,2,2),(2,2,3,3,2,2)$ & \\
for $\mathrm{k}=2, \mathrm{~m}=0.2$ & $\mathrm{ML}$ \\
$\left(\alpha_{1}, \alpha_{2}, \beta_{1}, \beta_{2}, \mu_{1}, \mu_{2}\right)=(2,2,3,3,2,2),(2,2,2,2,3,3)$ & \\
for $\mathrm{k}=1.6, \mathrm{~m}=0.4$ and & \\
$\left(\alpha_{1}, \alpha_{2}, \beta_{1}, \beta_{2}, \mu_{1}, \mu_{2}\right)=(2,2,2,2,3,3),(4,4,3,3,3,3)$ & \\
for $\mathrm{k}=1.1, \mathrm{~m}=0.9$ & \\
\hline
\end{tabular}




\section{References}

[1] Gogoi .J and Borah. M (2012), Estimation of Reliability for Multicomponent Systems Using Exponential Gamma and Lindley StressStrength Distributions, Journal of Reliability and Statistical Studies, Vol. 5, PP. 33-41.

[2] Mutkekar. R. R and Surekha B. Munoli. (2016), Estimation of Reliability for Stress-Strength Cascade Model, Open Journal of Statistics, Volume 6, PP .873-881.

[3] Sandhya. K and Uma Maheswari. T. S (2013), Reliability of a multi component stress strength model with standby system using mixture of two exponential distributions, Journal of Reliability and Statistical Studies, Vol. 6, PP 105-113

[4] Singh. D (2013), Measuring Reliability of n-Cascade System under random stress attention, International Journal of Scientific \& Engineering Research, Volume 4, PP 1400-1404

[5] Sundar. T. S (2012), Cascade Reliability of Stress-Strength system when Strength follows mixed Exponential distribution, International Journal of Engineering and Innovative Technolo-gy (IJEIT), Volume 1, PP. 103-110.

[6] Umamaheswari. T. S and Swathi. N (2013), Cascade Reliability of Stress-Strength system when Strength follows mixed Exponential distri-bution, IOSR Journal of Mathematics, Volume 4, Issue 5, PP.27-31.

[7] Umamaheswari. T. S and .Swathi. N (2013).Cascade Reliability for Generalized Expo-nential Distribution, International Journal of Computational Engineering Research, Vol. 3, Issue. 1 ,PP .132-136 